\title{
Effect of Platelet-rich Plasma on Mankin Scoring in Chemically-induced Animal Model of Osteoarthritis
}

\author{
Rafia Asjid1, Tayyaba Faisal1, Khadija Qamar1, Sana Malik2, Faiza Umbreen1 and Mahjabeen Fatima1 \\ ${ }^{1}$ Department of Anatomy, Army Medical College, National University of Medical Sciences, Rawalpindi, Pakistan \\ ${ }^{2}$ Department of Anatomy, Federal Medical and Dental College, Islamabad
}

\begin{abstract}
Objective: To evaluate the effects of platelet-rich plasma injection on histological changes of osteoarthritis using the modified Mankin score in monoiodoacetate-induced rat knee model.

Study Design: Laboratory-based experimental study.

Place and Duration of Study: Department of Anatomy, Army Medical College, Rawalpindi and National Institute of Health (NIH), Islamabad from March to May 2018.

Methodology: Thirty-two pathogen-free male rats aged 3-4 months were selected and divided into two groups with 8 and 24 animals, respectively. Groups A was used as control. Experimental group B was further subgrouped as B1, B2 and B3 with 8 animals, in each subgroup. Monoiodoacetate solution was injected into the articular cavity of the right knee joint of all the animals in group B. Both the control (A) and experimental group B1 were sacrificed after 2 weeks of MIA injection, for observing the histological changes in the articular cartilage. Group B2 received a single injection of platelet rich plasma (PRP) while group B3 was reared as such. Both groups were sacrificed after 4 weeks of intra-articular administration of PRP. Articular cartilage was collected, processed and stained with hematoxylin and eosin (H\&E) and toludine blue (T.B). Routine histological study was done and Mankin score was calculated to determine the grade of arthritis. SPSS Version 21 was used for the statistical analysis and p-value was considered significant at the cut-off value of 0.05 .

Results: The mean Mankin score of group B3 (9.37) was significantly higher than that of group B2 (7.37, p=0.003).

Conclusion: Platelet-rich plasma treatment controls the histological changes of chemically-induced osteoarthritis in rat knee.
\end{abstract}

Key Words: Osteoarthritis, Platelet-rich plasma, Mankin score.

How to cite this article: Asjid R, Faisal T, Qamar K, Malik S, Umbreen F, Fatima M. Effect of platelet-rich plasma on Mankin scoring in chemically-induced animal model of osteoarthritis. J Coll Physicians Surg Pak 2019; 29(11):1067-71.

\section{INTRODUCTION}

Osteoarthritis $(O A)$, is a progressive variety of arthritis affecting different joints of the body and one of the main causes of restriction of routine life activities. According to community-oriented programme for control of rheumatic diseases (COPCORD) studies conducted in Pakistan in $1998,3.7 \%$ of the 2,090 subjects were found to be suffering from knee OA. ${ }^{1}$ Features of $\mathrm{OA}$ include progressive cartilage degradation, subchondral bone remodelling, synovitis, and chronic pain.

Intra-articular administration of monoiodoacetate (MIA) has become a standard for induction of knee OA in rats and mice. ${ }^{2} \mathrm{MIA}$ decreases the sulphated proteoglycan content of the matrix and blocks the glycolytic pathway by interfering with the activity of glyceraldehyde-3phosphate dehydrogenase. ${ }^{3}$ Amount of MIA injected and the interval between the administration and observation of results affect the severity of arthritis.

Correspondence to: Dr. Rafia Asjid, Department of Anatomy,

Army Medical College, National University of Medical Sciences,

Rawalpindi, Pakistan

E-mail: rafiyainam@gmail.com

Received: February 21, 2019; Revised: July 03, 2019; Accepted: July 03, 2019
Pharmacological agents, knee debridement, osteotomy, and knee replacement surgeries are used for treatment of OA. Non-steroidal anti-inflammatory drugs, opioids, and disease modifying agents have been used since long for symptomatic relief in OA; but use of these drugs is associated with adverse side effects. Recently, platelet rich plasma (PRP) is a new advancement in treatment of OA. PRP is gaining significant attention as an autogenous source of growth factors like transforming growth factors (TGFs), connective tissue growth factors (CTGFs), insulin-like growth factors (IGFs), platelet derived growth factors (PDGFs), and fibroblast growth factors (EGFs \& FGFs), which are believed to influence the healing of the musculoskeletal system by promoting stem cells multiplication, collagen fibers synthesis and vasculogenesis. The beneficial effects of PRP are attributed mainly to TGF- $\beta$ which is involved in articular cartilage repair, matrix synthesis and cell proliferation. ${ }^{4}$ Various studies have been carried out to determine the concentration of platelets, which can be used for healing of musculoskeletal injuries.5,6

Spakova and colleagues compared the effects of PRP and hyaluronic acid and concluded that the mean Osteoarthritis index was improved in the PRP treated 
group. ${ }^{7}$ Gosen and colleagues carried out a randomised control trial for comparison of efficacy of corticosteroids and PRP injections in treatment of epicondylitis and concluded that PRP was found to be more effective for pain relief and improved function of the affected joint. 8 Several studies have been carried out to evaluate the in vitro effects of PRP, which supported its role in osteogenic activity of osteoblasts and chondrocytes in addition to fibroblast migration; 9,10 however, literature search indicated that PRP therapy only addresses the symptoms and does not affect the cartilage structure or progression of disease. This study was, therefore, carried out to establish the effects of platelet therapy on the histological changes occurring in the articular cartilage of MIA-induced model of osteoarthritis.

\section{METHODOLOGY}

It was a laboratory-based experimental study conducted at Department of Anatomy, Army Medical College, Rawalpindi and National Institute of Health (NIH), Islamabad, from March to May 2018.

All ethical aspects of this study were reviewed and approved by the Ethical Review Committee of Army Medical College, Rawalpindi.

Thirty-two adult healthy Sprague Dawley rats (purchased from NIH, Islamabad) aged 3 - 4 months, weighing 200300 gms were randomly selected using non-probability convenient sampling technique, and divided into two groups; control (A) and experimental (B). Experimental group $B$ was further split into three subgroups (B1, B2 and B3) with 8 rats in each subgroup. All animals were housed in separate cages, which were well ventilated. Moreover, 12-hour light and dark cycles were maintained at room temperature of $20^{\circ}-26^{\circ} \mathrm{C}$. Animals were fed standard lab diet and water ad libitum. All animal procedures and housing was done following the guidelines of animal house of National Institute of Health (NIH), Islamabad.

OA was induced in the experimental group by administrating $50 \mu \mathrm{l}$ of MIA (StruChem San Diego, Labseeker Inc.) solution, which was prepared by dissolving the chemical in $0.9 \%$ normal saline. Total $2 \mathrm{mg}$ of the chemical was injected into the right knee joint of each animal. Control A and experimental group B1 were sacrificed at day 14 for establishing whether osteoarthritic changes had occurred or not. Group B2 was treated with PRP; whereas group B3 was left as such.

PRP was prepared under sterile conditions using a double centrifugation technique. Intra-cardiac blood (3 $\mathrm{ml})$ was collected in a syringe containing $0.05 \mathrm{ml}$ of $0.1 \mathrm{M}$ sodium citrate as an anti-coagulant. PRP extraction was done following the protocol established by Zhang and his fellows. After centrifuging at $500 \times \mathrm{g}$ for 10 minutes, the plasma and buffy coat were aspirated and centrifuged again at $2200 \times g$ for 10 minutes. Platelet poor supernatant was then aspirated leaving the rest as PRP. ${ }^{11}$ Platelets were activated by adding 10\% calcium chloride $(50 \mu \mathrm{l}), 12$ to $0.5 \mathrm{ml}$ of platelet-rich plasma, which was injected into the articular cavity of the knee joint. Insulin syringes were used for the administration of PRP and MIA.

Rats in groups B2 (treated) and B3 (non-treated) were euthanised four weeks after the injection of PRP, by chloroform inhalation. Tibia of each right knee was collected and gross appearance of the cartilage was noted. India ink stain was used for better visualisation of the chondral lesions. ${ }^{13}$ The specimen were then fixed in $10 \%$ formalin. After decalcification with $5 \%$ nitric acid, the specimen were embedded in paraffin and $5 \mu \mathrm{m}$ thick sections were cut using rotary microtome. H\&E stains along with toludine blue were used for staining of the slides. Histological assessment of slides was done using modified Mankin score (Table I). Total score was calculated considering the parameters as structure of cartilage, cells count and TB staining of matrix. Hypercellularity/hypocellularity was labelled, if the cell count was above or below the normal range of cells in control group. Cloning was said to be present when a cluster of more than four chondrocytes was observed.14 Mankin scoring system was modified omitting the changes occurring in the tide mark. ${ }^{15}$ OA was classified as low, moderate or severe, if the score range was (1-3 points), (4-7 points) and (>8 points), respectively. ${ }^{16}$

SPSS Version 21 was used for analysis of data. Mankin score was expressed as mean \pm standard deviation (mean \pm S.D). Independant sample t-test was used to calculate the statistical significance among the treated versus the non-treated group. Keeping 95\% confidence level, statistical significance was present when the p-value was equal to or less than 0.05 .

\section{RESULTS}

Macroscopically, group B2 showed deep lesions in the cartilage surface with bony fragmentation. The gross changes were more severe in the non-treated group with wide spread areas of damage exposing the underlying bone. Microanatomic appearance of tissues among the groups B2 and B3 showed presence of osteoarthritic changes. In experimental group B2, surface fissures along with decrease in uncalcified cartilage thickness were noted (Figure 1). Cloning was seen in some animals with moderate decrease in staining of the matrix. The changes in the non-treated group B3 were of higher degree than those noted in the treated group B2. In the non-treated group, there was marked loss of cartilage height, extensive chondrocyte apoptosis along with severe depletion of the cartilage matrix. Multifocal collapse and erosion of subchondral bone was noted in some animals with bony outgrowths noted at the margins of the cartilage. The mean Mankin scores for 
Table I: Modified Mankin score.

\begin{tabular}{ll}
\hline Structure & 0 \\
Intact & 1 \\
Irregular surface & 2 \\
Fissures to transitional zone & 3 \\
Fissures to the deep zone & 4 \\
Complete disorganisation & 0 \\
\hline Cell count & 1 \\
Normal & 2 \\
Cloning & 3 \\
Hypercellularity & \\
Hypocellularity & 0 \\
Staining & 1 \\
Normal & 2 \\
Slightly reduced & 3 \\
Moderately reduced & 4 \\
Severe reduction & \\
Absence of dye &
\end{tabular}

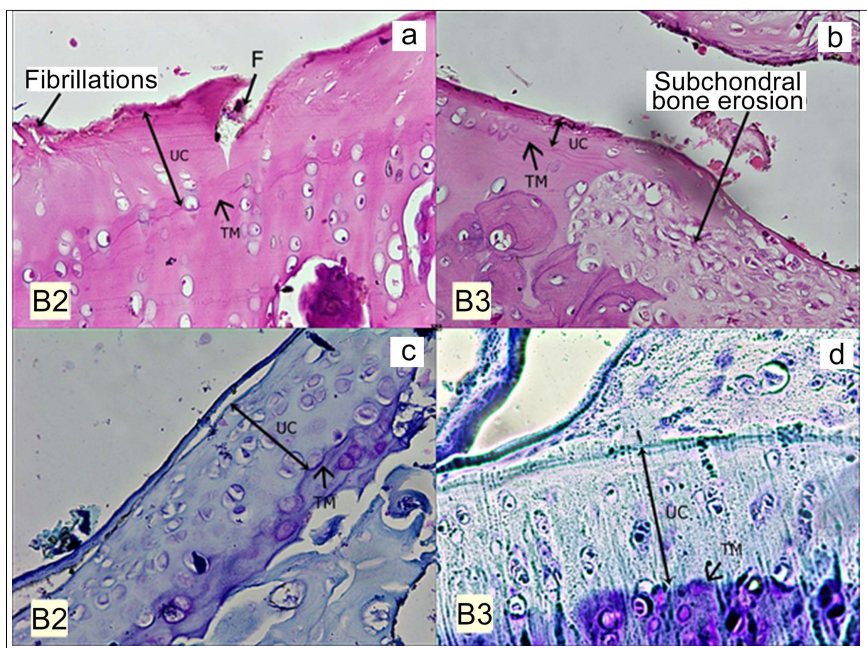

Figure 1: (a) articular cartilage of treated group (b) showing fibrillations, fissures (F) and empty lacunae (H\&E x 40). (b) showing subchondral bone erosion and decreased cartilage thickness in the non-treated group (B3) (H\&E x 40). (c \& d) Toluidine blue stained slide showing decreased matrix staining in treated (c) and non-treated (d) groups (x 40)

TM=Tidemark, UC=Uncalcified cartilage.

group B2 and B3 were $7.37 \pm 1.40$ (range 5-9) and 9.37 \pm 0.744 (8-10), respectively. Intergroup comparison of mean Mankin scores between the treated group B2 and the non-treated group B3 yielded a p-value of 0.003 , which was found to be statistically significant.

\section{DISCUSSION}

The present study was aimed to study the histological effects of autologous PRP injections in chemically induced osteoarthritic model of rat knee. Researches carried out on the effects of PRP showed favourable effects in clinical cases of $O A ; 17$ however, no significant work has been done to observe its effects on the histology of the affected cartilage. To the best of our knowledge, this is the first study evaluating the effects in chemical models of OA.

The potential of PRP to heal wounds and tendinopathies has been shown to have promising results. Laver and his colleagues published a systemic review on the role of PRP in clinical cases of knee and hip OA; and they concluded that clinical improvement was noted in PRPtreated groups as compared to the alternative treatment modalities. ${ }^{18}$ Many researches have shown that PRP starts to release its growth factors within the first 10 minutes, while releasing $95 \%$ within an hour. The role of platelets in maintenance of hemostasis and healing of wounds is attributed to the release of these factors. During the present study, double-spin concept was used to produce $0.5 \mathrm{ml}$ of liquid PRP producing platelet count, which was thrice that of baseline.

In this study, PRP prevented apoptosis of articular chondrocytes, which are responsible for secreting the cartilage matrix and maintaining the normal architecture of the cartilage. During this study, we successfully established an animal model of knee osteoarthritis, evident as significantly different mean Mankin scores in groups A and B1. Among groups B2 and B3, PRP therapy ameliorated the effect of MIA and caused the mean Mankin score to be less as compared to that of group B3, which did not receive platelet therapy. This finding was in accordance with the work carried out by Wu and colleagues, who observed the effects of PRP in surgical models of $\mathrm{OA}$ and found that the animals receiving PRP had less severe pathological changes as opposed to the group, which did not receive platelet injections. ${ }^{19}$ The present work was also supported by Patel and others, which showed that PRP injections improved WOMAC parameters (pain, stiffness and physical activity) in knee OA.20 Cole and others also carried out a randomised control trial for comparing the effects of PRP and HA and concluded that synovial fluid of the PRP-treated group showed lower concentrations of pro-inflammatory cytokines; IL-1 $\beta$ and TNF- $\alpha$ as compared to the ones receiving HA.21 Yun and fellows, compared the effects of mesenchymal stem cells and PRP on surgical model of OA in dogs and concluded that the PRP treated group had less mean Mankin score as compared to the stem cell-treated group,22 which augments our results. Kraeutler and his fellow workers reviewed the works carried out on the role of PRP and concluded that it has positive role in in vitro chondrocyte differentiation and enhanced cartilage repair in animal models of chondropathies. ${ }^{10}$

In summary, the present study concludes that platelet therapy was able to provide a protective effect by inhibiting the process of articular damage. Future research investigating the effects of PRP on other joints using multiple injections is required to establish the effect in this model.

The generalisation of this study results is limited by the fact that Mankin's histological histochemical grading system (MHHGS) has been critiqued for its validity in 
reproducing osteoarthritic changes in cartilage. Saal and Wyatt along with other researchers concluded that this scoring system does not allow to distinguish between mild and moderate grades of $O A ; 23$ however, it is reliable for differentiating normal and cartilage affected by high grade OA. Pritzker and his fellows concluded that faulty technique of slide preparation can be the cause for the non-reliability of this grading system. ${ }^{24}$

\section{CONCLUSION}

Platelet-rich plasma infusion ameliorates the effects of monoiodoacetate solution and prevents further deterioration of the cartilage structure as manifested by the mean Mankin score, which lead us to the conclusion that PRP has beneficial role in treating OA.

\section{FUNDING:}

This project was funded by National University of Medical Sciences (NUMS).

\section{DISCLOSURE:}

This article is based on the research thesis of one of the authors.

\section{ETHICAL APPROVAL:}

All ethical aspects of this study were reviewed and approved by the Ethical Review Committee of Army Medical College, Rawalpindi.

\section{CONFLICT OF INTEREST:}

Authors declared no conflict of interest.

\section{AUTHORS' CONTRIBUTION:}

RA: Designing of research project, collection, analysis and interpretation of data.

TF: Collection, analysis and interpretation of data.

$K Q$ : Conception of idea and final approval.

FU: Data analysis and critical review of article.

SM: Data collection.

MF: Designing of project.

\section{REFERENCES}

1. Haq SA, Davatchi F. Osteoarthritis of the knees in the copcord world. Int J Rheum Dis 2011; 14:122-9.

2. Pitcher $T$, Sousa-Valente J, Malcangio $M$. The mono-doacetate model of osteoarthritis pain in the mouse. $J$ Vis Exp 2016; 111.

3. Udo M, Muneta T, Tsuji K, Ozeki N, Nakagawa Y, Ohara T, et al. Monoiodoacetic acid induces arthritis and synovitis in rats in a dose and time-dependent manner: proposed model-specific scoring systems. Osteoarthritis Cartilage 2016; 24:1284-91.

4. Ashkavand Z, Malekinejad H, Vishwanath BS. The pathophysiology of osteoarthritis. J Pharm Res 2013; 7:132-8.

5. Dohan Ehrenfest DM, Andia I, Zumstein MA, Zhang CQ, Pinto NR, Bielecki T. Classification of platelet concentrates (platelet-rich plasma-Prp, platelet-rich fibrin-Prf) for topical and infiltrative use in orthopedic and sports medicine: Current consensus, clinical implications and perspectives. Muscles Ligaments Tendons J 2014; 4:3-9.

6. Fortier LA, Barker JU, Strauss EJ, McCarrel TM, Cole BJ. The role of growth factors in cartilage repair. Clin Orthop Relat Res 2011; 469:2706-15.

7. Spaková T, Rosocha J, Lacko M, Harvanová D, Gharaibeh A. Treatment of knee joint osteoarthritis with autologous plateletrich plasma in comparison with hyaluronic acid. Am J Phys Med Rehabil 2012; 91:411-7.

8. Gosens T, Peerbooms JC, van Laar W, den Oudsten BL. Ongoing positive effect of platelet-rich plasma versus corticosteroid injection in lateral epicondylitis: A double-blind randomized controlled trial with 2-year follow-up. Am J Sports Med 2011; 39:1200-8.

9. Mifune $\mathrm{Y}$, Matsumoto T, Takayama K, Ota S, Li H, Meszaros LB, et al. The effect of platelet-rich plasma on the regenerative therapy of muscle-derived stem cells for articular cartilage repair. Osteoarthritis Cartilage 2013; 21:175-85.

10. Kraeutler MJ, Chahla J, LaPrade RF, Pascual-Garrido C. Biologic options for articular cartilage wear (platelet-rich plasma, stem cells, bone marrow aspirate concentrate). Clin Sports Med 2017; 36:457-68.

11. Zhang J, Yuan T, Zheng N, Zhou Y, Hogan M, Wang JH. The combined use of kartogenin and platelet-rich plasma promotes fibrocartilage formation in the wounded rat achilles tendon entheses. Bone Joint Res 2017; 6:231-44.

12. Messora MR, Nagata MJH, Furlaneto FAC, Dornelles RCM, Bomfim SRM, Deliberador TM, et al. A standardized research protocol for platelet-rich plasma (Prp) preparation in rats. RSBO (Online) 2011; 8:299-304.

13. Schmitz N, Laverty S, Kraus V, Aigner T. Basic methods in histopathology of joint tissues. Osteoarthritis Cartilage 2010; 18:S113-S6.

14. Saal A, Gaertner J, Kuehling M, Swoboda B, Klug S. Macroscopic and radiological grading of osteoarthritis correlates inadequately with cartilage height and histologically demonstrable damage to cartilage structure. Rheumatol Int 2005; 25:161-8.

15. Thomas C, Fuller C, Whittles C, Sharif M. Chondrocyte death by apoptosis is associated with cartilage matrix degradation. Osteoarthritis Cartilage 2007; 15:27-34.

16. Miyamoto S, Nakamura J, Ohtori S, Orita S, Omae T, Nakajima T, et al. Intra-articular injection of mono-lodoacetate induces osteoarthritis of the hip in rats. BMC Musculoskelet Disord 2016; 17:132.

17. Meheux CJ, McCulloch PC, Lintner DM, Varner KE, Harris JD. Efficacy of intra-articular platelet-rich plasma injections in knee osteoarthritis: A systematic review. Arthroscopy 2016; 32: 495-505.

18. Laver L, Marom N, Dnyanesh L, Mei-Dan O, EspregueiraMendes J, Gobbi A. Prp for degenerative cartilage disease: A systematic review of clinical studies. Cartilage 2017; 8:341-64.

19. Wu J, Zhang Z, Qin X, Cai X, Hu F. Platelet-rich-plasma alleviates pathological symptoms in a rabbit model of osteoarthritis. Int $J$ Clin Exp Med 2016; 9:21038-47.

20. Patel S, Dhillon MS, Aggarwal S, Marwaha N, Jain A. Treatment 
with Platelet-rich plasma is more effective than placebo for knee osteoarthritis: A prospective, double-blind, randomized trial. Am J Sports Med 2013; 41:356-64.

21. Cole BJ, Karas V, Hussey K, Merkow DB, Pilz K, Fortier LA. Hyaluronic acid versus platelet-rich plasma: A prospective, double-blind randomized controlled trial comparing clinical outcomes and effects on intra-articular biology for the treatment of knee osteoarthritis. Am J Sports Med 2017; 45:339-46.

22. Yun S, Ku SK, Kwon YS. Adipose-derived mesenchymal stem cells and platelet-rich plasma synergistically ameliorate the surgical-induced osteoarthritis in beagle dogs. J Orthop Surg Res 2016; 11:9.

23. Wyatt LA, Moreton BJ, Mapp PI, Wilson D, Hill R, Ferguson E, et al. Histopathological subgroups in knee osteoarthritis. osteoarthritis cartilage $2017 ; \mathbf{2 5 : 1 4 - 2 2}$.

24. Pritzker KPH, Gay S, Jimenez SA, Ostergaard K, Pelletier JP, Revell PA, et al. Osteoarthritis cartilage histopathology: Grading and staging. Osteoarthritis Cartilage 2006; 14:13-29.

…두.... 